\title{
Augmented Reality Training for Assembly and Maintenance Skills
}

\author{
Sabine Webel* \\ Uli Bockholt* \\ Timo Engelke* \\ Matteo Peveri*† \\ Manuel Olbrich* \\ Carsten Preuscheł \\ (*)Fraunhofer IGD, Germany, \\ $\left({ }^{*}+\right.$ ) Sidel S.p.A, Italy \\ (†)German Aerospace Center (DLR), Institute of Robotics and Mechatronics, Germany \\ E-mail: Sabine.Webel@igd.fraunhofer.de,Ulrich.Bockholt@igd.fraunhofer.de, \\ Timo.Engelke@igd.fraunhofer.de,Matteo.Peveri@sidel.com, \\ Manuel.Olbrich@igd.fraunhofer.de,Carsten.Preusche@dlr.de
}

\begin{abstract}
Augmented Reality (AR) points out to be a good technology for training in the field of maintenance and assembly, as instructions or rather location-dependent information can be directly linked and/or attached to physical objects. Since objects to maintain usually contain a large number of similar components (e.g. screws, plugs, etc.) the provision of location-dependent information is vitally important. Another advantage is that AR-based training takes place with the real physical devices of the training scenario. Thus, the trainee also practices the real use of the tools whereby the corresponding sensorimotor skills are trained.
\end{abstract}

\section{Introduction}

Actual developments in ICT technologies are driven by two trends fostering fundamental paradigm changes in interaction and information processing. On the one hand smartphone and mobile computing technologies offer high sophisticated platforms integrating multimodal sensory and powerful CPUs, on the other hand information is structured geo-referenced linking information to specific locations within our environment. Thereby, Augmented Reality (AR) has become a key technology as it analyses the sensor data (camera, GPS, inertial) to derive the detailed pose of the mobile system, with the aim to correlate our real environment to the geo-referenced information space. Thus, AR realizes a camera based interaction fusing Computer Vision with Computer Graphic.

\section{Related Work}

As the complexity of maintenance and assembly procedures can be enormous, the training of operators to perform those tasks efficiently has been in focus of many research groups. Numerous studies presented the potential of AR-based training systems and its use in guidance applications for maintenance tasks [1,2,3]. Tümler et al. [4] in this context focused on the question, how long term usage of AR-technology in industrial training and assisting applications produces stress and strain for the user. They showed that the use of optimal AR system (no lag, high quality see-through calibration, weight-reduced HMD etc.) decreases the overall strain, so that working with the AR system could mean a decreased strain compared to traditional work assistance systems.

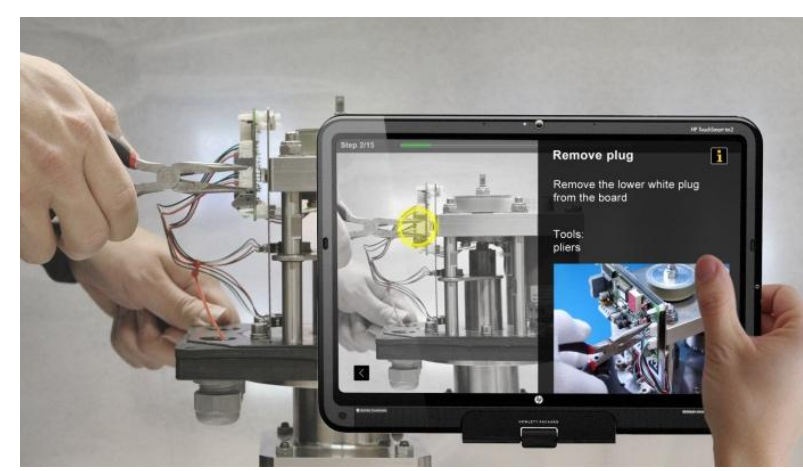

Figure 1: AR-Training of an Assembly Task using "Virtual Post-Its"

The work of Franklin [5] focuses on the application of AR in the context of Forward Air Controller training. The author concluded that the impact of AR for training depends on the specific requirements of the end user and in particular on the realism of the stimulation required. An approach in the context of robot Programming-by-Demonstration is presented in [6]. The authors define learning accelerators, which are implemented and evaluated in AR setups and present a

This is an Open Access article distributed under the terms of the Creative Commons Attribution-Noncommercial License 3.0, which permits unrestricted use, distribution, and reproduction in any noncommercial medium, provided the original work is properly cited. 
training protocol for training Programming-byDemonstration skills. Also another work showed the potential of $\mathrm{AR}$ in the context of human-robot interaction for industrial robots [7]. It has been pointed out that handhelds and monitor based visualization devices are better suited for industrial environments than Head Mounted Displays, since they are more robust.

\section{AR and Teleconsultation}

AR is used for skill transfer in industrial training addressing assembly and maintenance of large machineries and plants integrating capturing and rendering technologies (cf. Figure 1):

- Capturing: The camera is used to capture activities (of an expert) within a large scaled environment. Thereby, considering the different modalities registered the captured activities can be represented in different qualities. "Virtual PostIts" are linking contextual information to specific machine parts. Starting with forces/torques to be applied within an assembly task and resulting in multimedial illustrations including audio/video files the "Virtual Post-Its" can document complex assembly or maintenance workflows.
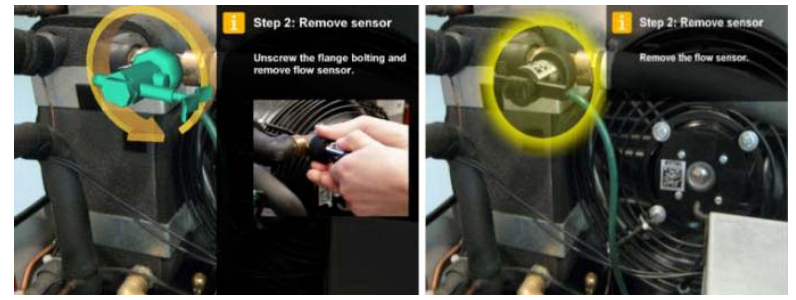

Figure 1: Adaptive Visual Aid with activated content object (a) at a strong guidance level and (b) at a softer guidance level

- Rendering: Within the training scenario the captured information can be presented in two different modalities: Either a 3D-animation illustrating the current assembly step is directly superimposed to the captured video image ("Direct Visual Aid"), or the tracked "Virtual Post-It" icon indicates that contextual information is available for a machine part to be displayed if the icon is selected by the user ("Indirect Visual Aid"). In this way the guidance level can be adapted to the trainee's pre-knowledge realizing different guiding levels (cf. Figure 1).

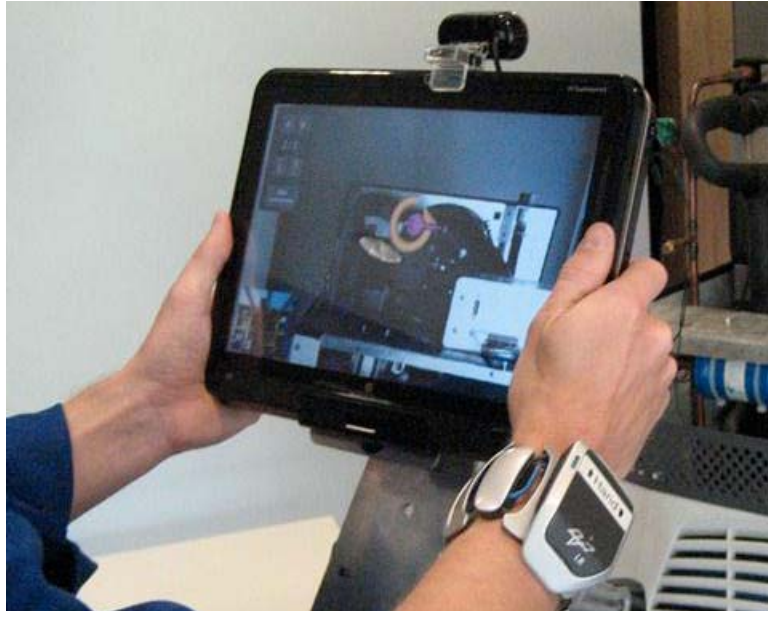

Figure 2: Mobile AR component including haptic bracelet

This approach for "Skills transfer" in industrial training offers high impact as it can be integrated in teleconsultation scenarios in the following way:

- The trainee users the mobile AR-Equipment to inspect the machinery to be operated (cf. Figure 2 ). Thereby, step by step he/she is guided through the training procedure. If a problem occurs during the training session he/she connects to the remote trainer via UMTS of WIFI.

- The video images captured with the mobile system of the trainee are now transferred in real-time to the trainer. Thus, the trainer is able to reproduce the problem of the trainee and he is able to modify or to enhance the training protocol. Therefore he/she adds new "Virtual Post-Its" into the training protocol that can integrate scribbled annotations to the captured video images (cf. Figure 4). The trainer can also link additional Multi-media illustrations to the addressed assembly step. In this way the teleconsultation component is also used as Augmented Reality authoring system: All the annotations added by the remote trainer are integrated into the training protocol and they are linked to the tracked machine parts.

\section{Haptic Hints}

In maintenance and assembly tasks the operator acts mainly with his hands. Thus, the application of haptic feedback to the trainee's hands during the training of those tasks can support him in comprehending how to perform the task and in performing it. Devices like vibrotactile bracelets offer the possibility to apply 
vibration stimuli to the human arm, forearm, and wrist (cf. Figure 3). Those bracelets are equipped with six vibration actuators which are placed at equal distance from each other inside the bracelet and thus also around the user's arm. The intensity of each actuator can be controlled individually. That way, various sensations can be generated such as rotational or translational movement hints. Usually a lot of visual information has to be processed in complex working scenarios. In contrast, the tactile channel is less overloaded. Furthermore, vibrotactile feedback is a quite intuitive feedback, as the stimuli are directly mapped to body coordinates.

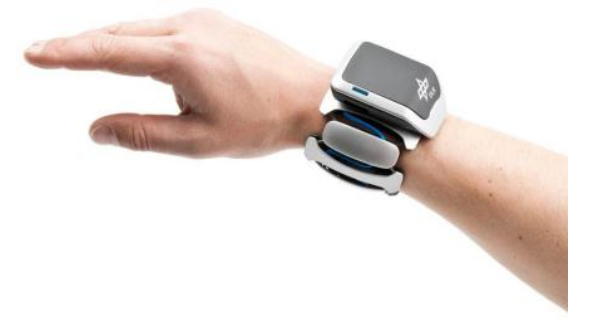

Figure 3: Vibrotactile bracelet

Since it provides a soft guidance which "channels" the user to the designated target instead of directly manipulating his movements, it does not prevent the active exploration of the task. In this sense vibrotactile feedback is used to give the trainee additional movement hints, such as rotational or translational movements cues, and to guide the trainee to specific targets. For example, if the trainee needs to rotate his arm for performing a sub-task, the rotational direction (clockwise or anti-clockwise) may be difficult to recognize in a video showing an expert performing the sub-task. Receiving the same information using a vibrotactile bracelet, the trainee can easier identify the rotational direction. Also translational movements can be conveyed using vibrotactile stimuli.

Apart from that also for presenting error feedback, such as communicating whether the right action is performed (e.g. the right tool is grasped), it is useful to apply vibrotactile feedback. This is a significant factor, as it can prevent the user from performing errors at an early stage. In addition, vibrotactile hints should be used to provide slight instructions by directing the trainee's attention to a body part and indicating movements.

\section{Augmented Reality based Training}

The great advantage of using Augmented Reality for training is that the trainee can interact with the real world objects and simultaneously access the virtual information for guidance. Therefore, the trainee can easily accomplish the mapping between the training and the real task. Furthermore, he/she can perform the actual task while accessing additional training material, and that way learning is facilitated. With regard to Fitt's model of skill acquisition [8] this denotes that AR enables the trainee to learn the basics about the task by observing the augmented instructions and trying to perform the instructed sub-task, to develop behavior and movement patterns when performing the sub-tasks, and to redefine those motor patterns in repeated performances of the task (i.e. during training). By accessing augmentations while the trainee is performing the task training, he becomes partially skilled. Hence, the trainee's skill level starts to develop when he performs the real task for the first time.

Another advantage of AR-based training is that the trainee has real tactile feedback when performing the training task, since he can interact with real objects. The virtual objects provide additional information about the task and its performance and supplement the trainee's knowledge about the task. Accordingly, the trainee can access the training material (i.e. the virtual instructions, etc.) and the real environment without the need to use "external" separate training material (e.g. a user manual).

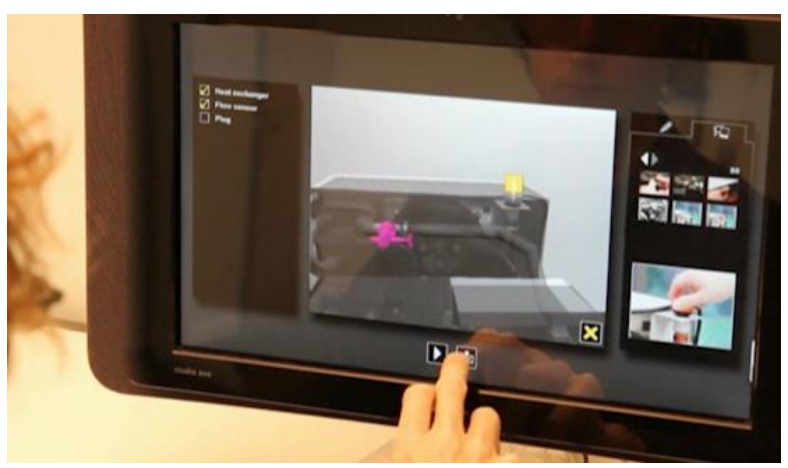

Figure 4: Authoring/Teleconsultation Tool for the specification of the training protocol

Furthermore, the use of a training platform that involves virtual elements - like for instance an Augmented Reality platform - allows for the measurement and evaluation of the trainees performance in modes and levels of detail that are not possible when performing the actual task in real world without using virtual components. By involving a technology providing virtual elements, it is also possible to respond to the trainee's performance and present corresponding feedback in a way that is not available without this technology. In addition, the type and order of presented sub-tasks can be adapted, which is not possible in the real world. 
A potential danger of $A R$ applications is that users become dependent on AR features such as visual instructions. As a result, the user might not be able to perform the task when those features are not available or when the technology fails. This leads to two demands on AR training applications and programs: On the one hand, the training programs should include phases in which the amount of AR features is reduced (less virtual components, e.g. only instructions for the current sub-task without additional information about the device, tools, etc.). On the other hand, the training program should also include phases in which the level of information provided by the AR features is reduced (e.g. only spatial hints without detailed instructions). That is, the level of guidance in the training system has to be adaptable to the current training phase. To summarize, AR-based training applications must clearly differ from AR-based guiding applications, as they must really train the user and not only guide him through the task. This can be only achieved by involving cognitive aspects in the training.

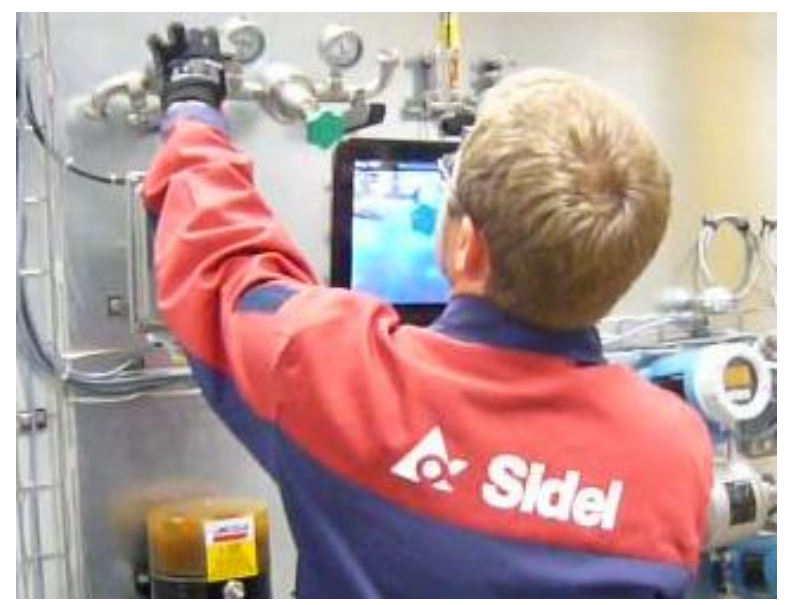

Figure 5: Evaluation of AR-based Training at Sidel training center for service technicians

\section{Conclusions and Acknowledgments}

AR offers high potential for industrial training, as it can be integrated in teleconsultation technologies and it can be realized on standard hardware systems like smartphones. The use of AR-based training technologies has been evaluated in the context of training of complex maintenance procedures in machine building. Experienced service technicians utilized AR technologies to receive information about the maintenance task to perform (cf. Figure 5).

After this AR-based Training, they performed the same task without any aids. In this control step, the technicians' performance (number of errors, performance time) has been measured and compared to the performance of technicians who trained the task using traditional training methods.

The activities described in this paper have been carried out with funding of the EU within the integrated project SKILLS "Multimodal Interfaces for capturing and Transfer of Skill"- IST-2006-035005 (www.skills-ip.eu). Authors are grateful to the EU for the support given in carrying on these activities. The AR platform development has been done in close cooperation with all SKILLS partners in particular with the colleagues of PERCRO, DLR, LABEIN, CEIT, TECHNION, Aalborg University, OMG, KUKA SIDEL, Queens University Belfast, University of Montpellier, CEA-List, Haption, OMG and PERCRO.

\section{References}

[1] B. Schwald, B. Laval, T. Sa, R. Guynemer. An Augmented Reality system for training and assistance to maintenance in the industrial context. In Proceedings of the 11th International Conference in Central Europe on Computer Graphics, Visualization and Computer Vision (WSCG '03), pp. 425-432. 7, 2003

[2] A. Olwal, J. Gustaffsson, C. Linfors. Spatial Augmented Reality on industrial CNC-machines. In Proceedings of the SPIE: The Engineering Reality of Virtual Reality, vol. 6804, pp. 680409.1-680409.9. 8, 2008

[3] C. Ke, B. Kang, D. Ch3en, X. Li. An Augmented Reality-based application for equipment maintenance. In ACII, pp. 836-841. 8, 2005

[4] J. Tümler, F. Doil, R. Mecke, G. Paul, M. Schenk, E. Pfister, A. Huckauf, I. Bockelmann, A. Roggentin. Mobile Augmented Reality in industrial applications: Approaches for solution of user-related issues. In Proceedings of the 7th IEEE and ACM International Symposium on Mixed and Augmented Reality (ISMAR '08), IEEE Computer Society, pp. 87-90. 8, 2008

[5] M. Franklin. The lessons learned in the application of Augmented Reality. In Virtual Media for Military Applications, pp. 30/1-30/8. 9, 2006

[6] V. Schmirgel, U. Zimmermann, E. Yechiam, T. Hulin, C. Preusche. Comprehension of operating a robot by enactive learning: Examplary approaches with programming-by-demonstration. In Enaction on SKILLS: International Conference on Multimodal Interfaces for Skills Transfer (SKILLS '09), pp. 135142. 9, 2009

[7] R. Bischoff, A. Kazi. Perspectives on Augmented Reality based human-robot interaction with industrial robots. In Proceedings of the IEEE/RSJ International Conference on Intelligent Robots and Systems (IROS), vol. 4, pp. 3226-3231. 9, 2004

[8] S. MacKenzie. Fitts' law as a research and design tool in human-computer interaction. In Human-Computer Interaction, pp. 91-139. 7, 1992 